\title{
Implementation of Elastic Prestack Reverse-Time Migration Using an Efficient Finite-Difference Scheme
}

\author{
Hongyong YAN ${ }^{1,2}$, Lei YANG ${ }^{1,3}$, Hengchang $\mathrm{DAI}^{2}$, and Xiang-Yang $\mathrm{LI}^{2}$ \\ ${ }^{1}$ Key Laboratory of Petroleum Resources Research, \\ Institute of Geology and Geophysics, Chinese Academy of Sciences, Beijing, China \\ ${ }^{2}$ British Geological Survey, Murchison House, Edinburgh, United Kingdom \\ ${ }^{3}$ University of Chinese Academy of Sciences, Beijing, China \\ e-mail: yanhongyong@163.com (corresponding author)
}

\begin{abstract}
Elastic reverse-time migration (RTM) can reflect the underground elastic information more comprehensively than single-component Pwave migration. One of the most important requirements of elastic RTM is to solve wave equations. The imaging accuracy and efficiency of RTM depends heavily on the algorithms used for solving wave equations. In this paper, we propose an efficient staggered-grid finite-difference (SFD) scheme based on a sampling approximation method with adaptive variable difference operator lengths to implement elastic prestack RTM. Numerical dispersion analysis and wavefield extrapolation results show that the sampling approximation SFD scheme has greater accuracy than the conventional Taylor-series expansion SFD scheme. We also test the elastic RTM algorithm on theoretical models and a field data set, respectively. Experiments presented demonstrate that elastic RTM using the proposed SFD scheme can generate better images than that using the Taylor-series expansion SFD scheme, particularly for PS images. Fur-
\end{abstract}

Ownership: Institute of Geophysics, Polish Academy of Sciences

(C) 2016 Yan et al. This is an open access article distributed under the Creative Commons

Attribution-NonCommercial-NoDerivs license

http://creativecommons.org/licenses/by-nc-nd/3.0/. 
thermore, the application of adaptive variable difference operator lengths can effectively improve the computational efficiency of elastic RTM.

Key words: seismic imaging, elastic wave, wavefield extrapolation, finite-difference.

\section{INTRODUCTION}

Vector seismic exploration can reflect the underground elastic information more comprehensively than single P-wave exploration, so multicomponent seismic imaging has become an active research field for exploration geophysicists (Yan et al. 2013). Early attempts at multicomponent imaging used Kirchhoff migration. Elastic Kirchhoff migration has been implemented by Kuo and Dai (1984) and by Hokstad (2000), respectively. The elastic Kirchhoff migration is highly efficient. However, it suffers from drawbacks similar to those of acoustic Kirchhoff migration, because ray theory breaks down when subsurface structures are complex (Gray et al. 2001, Yan and Sava 2008). Reverse-time migration (RTM) based on directly solving the two-way wave equation has provided a superior way to image complex subsurface structures, and this seismic imaging technology has been applied successfully in scalar seismic exploration (e.g., Baysal et al. 1983, Whitmore 1983). The RTM based on vector wave field is becoming more and more important. Chang and McMechan $(1987,1994)$ successively implemented the 2D and 3D elastic RTM based on vector wave equations. In their elastic RTM scenario, the extrapolated wavefields are vectors, and P- and S-modes are mixed, and the images formed by direct crosscorrelation of source and receiver wavefields mix the contributions from P- and S-waves. The mixed PP and PS images lead to an unclear physical meaning, so the migrated vector sections are difficult to interpret.

To get images with clear physical meanings, it is preferred to separate Pand S-modes, and implement imaging conditions for pure wave modes (Yan and Xie 2012). Sun and McMechan (2001) proposed elastic RTM based on a scalar wave equation. Their procedure requires the separation of vector data into P- and S-wave components on the acquisition surface and then two RTMs of P- and S-waves based on a scalar wave equation can be performed independently. However, wavefield separation on the surface is not always satisfied, and this procedure neglects the vector characteristic of elastic waves. Yan and Sava (2008) advocated another procedure for imaging elastic wavefield data, which used entire vector wavefields for wavefield reconstruction and performed an additional wavefield separation before applying an imaging condition. This approach makes images cleaner and easier to interpret because they represent reflections of single wave modes at interfaces between different of physical properties, and it maintains the vector characteristic of elastic waves. Yan and Sava (2008) used this procedure to imple- 
ment RTM with angle-domain imaging formulated for multicomponent elastic data. Du et al. $(2012,2014)$ followed the procedure presented by Yan and Sava (2008) to perform elastic RTM in the common-shot domain and discussed the polarity reversal correction by using the energy flux density vector. Chung et al. (2012) proposed a frequency domain elastic RTM algorithm in the common shot domain based on the wavefield separation technology. Yan and Xie (2012) presented an angle-domain imaging condition using the local slant stack method for multicomponent elastic RTM.

Elastic RTM requires forward extrapolation of the source wavefield and backward extrapolation of the recorded receiver wavefield in time (Yan and Sava 2008), and the essence of wavefield extrapolation is the numerical solution of wave equations. The imaging accuracy and efficiency of elastic RTM depends heavily on the algorithms used for solving wave equations. Hence, how to solve wave equations is very important to the implementation of elastic RTM. In seismic exploration, the most popular implementation for solving equations in wavefield extrapolation is finite-difference (FD) methods, especially staggered-grid FD (SFD) methods (Liu et al. 2009, Liu and Sen 2011b, Yang et al. 2014). However, numerical approximations of the FD methods are required for spatial and time derivatives, and the conventional FD methods often suffer from serious numerical errors (numerical dispersion) (Li et al. 2013). Traditionally, accurate numerical approximations are achieved by using either relatively very fine computation grids or very long FD operators (Dablain 1986). Otherwise, numerical dispersion will be present in the image data, which contaminates the signals. However, either approach will increase the computational cost dramatically (Liu et al. 2009). Another alternative is to optimize the FD coefficients to suppress the numerical dispersion in seismic modeling and imaging (e.g., Li et al. 2015, Yang et al. 2015, Yan et al. 2015).

In this paper, we propose an efficient SFD scheme based on a sampling approximation method with adaptive variable difference operator lengths to implement elastic prestack RTM. First, we simply review the conventional SFD scheme based on Taylor-series expansion method, and introduce a high-accuracy SFD scheme based on a sampling approximation method and adaptive variable difference scheme. Then, we analyze the accuracy of the numerical solution of elastic wave equations using the SFD schemes, including the Taylor-series expansion SFD and sampling approximation SFD schemes. Next, we introduce the basic workflow of the elastic RTM. Finally, we adopt the SFD scheme based on the sampling approximation method with adaptive variable difference operator lengths to implement forward and backward wavefield extrapolations for elastic prestack RTM, testing the algorithm with two theoretical models and a field data set, respectively. 


\section{METHOD}

\subsection{SFD schemes for the numerical solutions of elastic wave equations}

In $2 \mathrm{D}$ heterogeneous media, the elastic velocity-stress equations are (Virieux 1986)

$$
\begin{gathered}
\frac{\partial v_{x}}{\partial t}=\frac{1}{\rho}\left(\frac{\partial \tau_{x x}}{\partial x}+\frac{\partial \tau_{x z}}{\partial z}\right), \\
\frac{\partial v_{z}}{\partial t}=\frac{1}{\rho}\left(\frac{\partial \tau_{x z}}{\partial x}+\frac{\partial \tau_{z z}}{\partial z}\right), \\
\frac{\partial \tau_{x x}}{\partial t}=(\lambda+2 \mu) \frac{\partial v_{x}}{\partial x}+\lambda \frac{\partial v_{z}}{\partial z}, \\
\frac{\partial \tau_{z z}}{\partial t}=\lambda \frac{\partial v_{x}}{\partial x}+(\lambda+2 \mu) \frac{\partial v_{z}}{\partial z}, \\
\frac{\partial \tau_{x z}}{\partial t}=\mu \frac{\partial v_{x}}{\partial z}+\mu \frac{\partial v_{z}}{\partial x},
\end{gathered}
$$

where $t$ is the time, $x$ and $z$ are the space coordinates, $\left(v_{x}, v_{z}\right)$ is the particle velocity vector, $\left(\tau_{x x}, \tau_{z z}, \tau_{x z}\right)$ is a vector containing three components of stress, $\rho(x, z)$, is the density, and $\lambda(x, z)$, and $\mu(x, z)$ are Lame's constants.

To solve the 2D elastic velocity-stress equations, the high-order SFD schemes are generally used to calculate the spatial first-order derivatives. The (2M)th-order SFD scheme for the first-order derivative of the function $p(x)$ can be expressed as (Kindelan et al. 1990)

$$
\frac{\partial p}{\partial x} \approx \frac{1}{h} \sum_{m=1}^{M} a_{m}[p(x+m h-0.5 h)-p(x-m h+0.5 h)],
$$

where $h$ is the grid size and $a_{m}$ are difference coefficients on the first-order derivative.

Using plane wave theory, we obtain:

$$
p(x+m h)=p_{0} \exp [i k(x+m h)],
$$

where $p_{0}$ is a constant, $i=\sqrt{-1}$, and $k$ is the wavenumber. Substituting Eq. 3 into Eq. 2, the dispersion relation can be obtained as follows (e.g., Yang et al. 2014, Yan et al. 2015):

$$
\beta \approx \sum_{m=1}^{M} a_{m} \sin [(2 m-1) \beta],
$$


where: $\beta=k h / 2$ and $0 \leq \beta \leq \pi / 2$.

When the Taylor-series expansion method (e.g., Dong et al. 2000, Pei 2004, Liu and Sen 2009) is employed to compute the SFD coefficients, Eq. 4 can be expanded as

$$
\beta \approx \sum_{n=1}^{\infty}\left[\frac{(-1)^{n-1}}{(2 n-1) !} \sum_{m=1}^{M}(2 m-1)^{2 n-1} a_{m} \beta^{2 n-1}\right] .
$$

Comparing the coefficients of $\beta$ in Eq. 5, we obtain the SFD coefficients based on Taylor-series expansion method as follows (Liu and Sen 2009):

$$
a_{m}=\frac{(-1)^{m+1}}{2 m-1} \prod_{1 \leq n \leq M, n \neq m}\left|\frac{(2 n-1)^{2}}{(2 m-1)^{2}-(2 n-1)^{2}}\right| .
$$

When the conventional classic coefficients determined by the Taylorseries expansion method are used to solve wave equations for a larger frequency content, serious numerical dispersion will occur, which will affect the seismic modeling accuracy and imaging quality (Yan et al. 2015).

We introduce a high-accuracy SFD scheme with weak-dispersion to solve elastic wave equations. First, we take $M$ sampling points for $\beta$, which can be expressed as $\beta_{1}, \beta_{2}, \ldots \beta_{M}$, and are distributed evenly over the range from 0 to a given $u$, where $u$ is a constant, and $u \in(0, \pi / 2]$. Then we approximate the dispersion relation (Eq. 4 ) at the $M$ sampling points. When $\beta_{1}=0$, the equality of Eq. 4 can be unconditional. The basic idea is similar to the optimization method of Yang et al. (2015), but their optimization method is for rotated SFD scheme. The near zero-wavenumber constraint condition is usually adopted to improve the accuracy of FD (e.g., Zhou and Zhang 2011, Liu 2014, Yang et al. 2015). We introduce the constraint condition by using the following formula:

$$
\lim _{\beta \rightarrow 0} \frac{\sum_{m=1}^{M} a_{m} \sin [(2 m-1) \beta]}{\beta}=1,
$$

Then, we obtain:

$$
a_{1}=1-\sum_{m=2}^{M} a_{m}(2 m-1) .
$$

Substituting Eq. 8 into Eq. 4, we obtain:

$$
\sum_{m=2}^{M}\{\sin [(2 m-1) \beta]-(2 m-1) \sin \beta\} a_{m} \approx \beta-\sin \beta .
$$



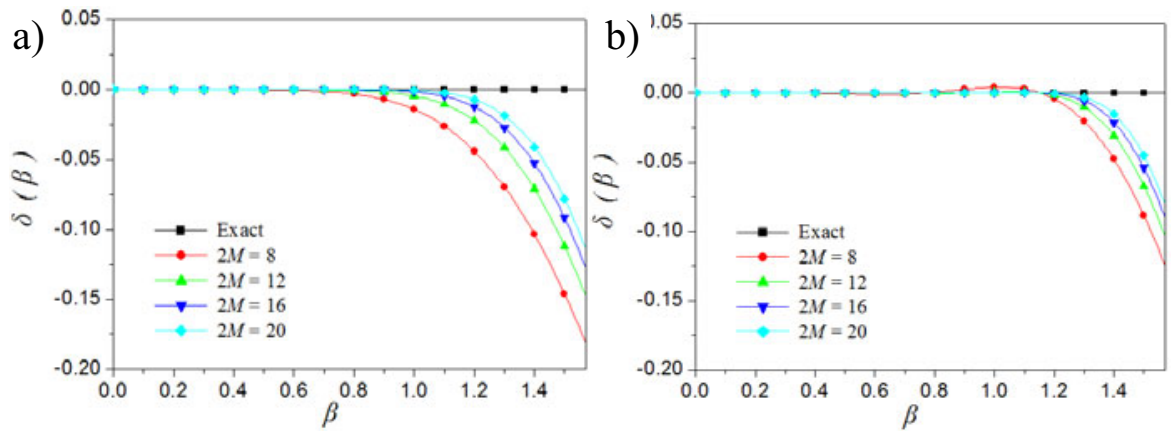

Fig. 1. Plot of numerical dispersion curves by (a) the Taylor-series expansion SFD scheme and (b) the sampling approximation SFD scheme for different operator lengths.

Subsequently, we can use the rest of the $(M-1)$ sampling points $\left(\beta_{1}, \beta_{2}\right.$, $\beta_{M}$ ) to construct a system of linear equations from Eq. 9, and then obtain the SFD coefficients for first-order derivatives by solving these linear equations and Eq. 8. The difference coefficients can be simply obtained by this method. We call the method sampling approximation SFD scheme.

According to Eq. 4, the parameter $\delta(\beta)$ is defined to describe the numerical dispersion in SFD modeling:

$$
\delta(\beta)=\frac{\sum_{m=1}^{M} a_{m} \sin [(2 m-1) \beta]}{\beta}-1 .
$$

If $\delta(\beta)$ is close to zero, the numerical dispersion is weak. When $\delta(\beta)$ is much greater or less than zero, large numerical dispersion will exist.

To compare the sampling approximation SFD with Taylor-series expansion SFD schemes, we analyze the numerical dispersion relations by showing the dispersion curves. Figure 1 shows variations of $\delta(\beta)$ with $\beta$ for different $M$ by the Taylor-series expansion SFD and the sampling approximation SFD for numerical dispersion, respectively. From Fig.1, we can see that the sampling approximation SFD scheme has greater accuracy than the Taylor-series expansion SFD scheme over a wider range of wavenumbers. Namely, the sampling approximation SFD scheme suffers much less numerical dispersion than the Taylor-series expansion SFD scheme for the same spatial difference operator length. In addition, the difference operator length of sampling approximation SFD scheme is shorter than that of Taylor-series expansion SFD scheme with the same numerical accuracy.

Generally, the fixed difference operator length is used to compute the spatial derivatives, which leads to more computing time. Based on the idea 
of the adaptive variable-length FD operators presented by Liu and Sen (2011a), we propose the sampling approximation SFD scheme with adaptive variable difference operator lengths. It uses long operators in regions of low velocity and short operators in regions of high velocity to improve the computational efficiency for the solution of elastic wave equations in elastic prestack RTM.

According to Eq. 10, the numerical error $\varepsilon$ is defined as follows:

$$
\varepsilon=\delta(\beta)=\frac{v \sum_{m=1}^{M} a_{m} \sin [(2 m-1) \pi f h / v]}{\pi f h}-1,
$$

where $f$ is the frequency and $v$ is velocity. Here, we adopt the S-wave velocity to calculate the numerical error because the S-wave dispersion is dominant in elastic wave propagation.

From Eq. 11, we find that $\varepsilon$ is a function of $v, M$ and $f$. Therefore, for the given maximum frequency $f_{\max }$ and maximum numerical error $\eta$, the following inequality is satisfied (e.g., Liu and Sen 2011a)

$$
|\varepsilon(v, M, f)| \leq \eta,
$$

where $f \leq f_{\max }$. The adaptive variable SFD operator lengths can be determined by the inequality (Eq. 12).

\subsection{The basic workflow of elastic prestack RTM}

The implementation of elastic prestack RTM consists of three main parts: forward extrapolation of the source wavefield in time, backward extrapolation of the recorded receiver wavefield in time, and the application of an imaging condition to the pure waves (Yan and Sava 2008). The workflow includes the same three typical parts found in acoustic RTM. However, more operations are performed. After reconstructing the source wavefield and the receiver wavefield, we should separate the elastic wavefields into P- and Swaves, and then perform the phase correction, the amplitude balancing and the polarity reversal correction prior to applying the imaging condition. Figure 2 is the workflow chart for elastic prestack RTM.

The extrapolated wavefield should be separated into P- and S-wave components after extrapolation and crosscorrelation of the vector and scalar potentials are used for imaging (Dellinger and Etgen 1990). In a 2D isotropic medium, the P- and S- wave components can be obtained by applying Helmholtz decomposition to the elastic extrapolated wavefield (e.g., Yan and Sava 2008). 


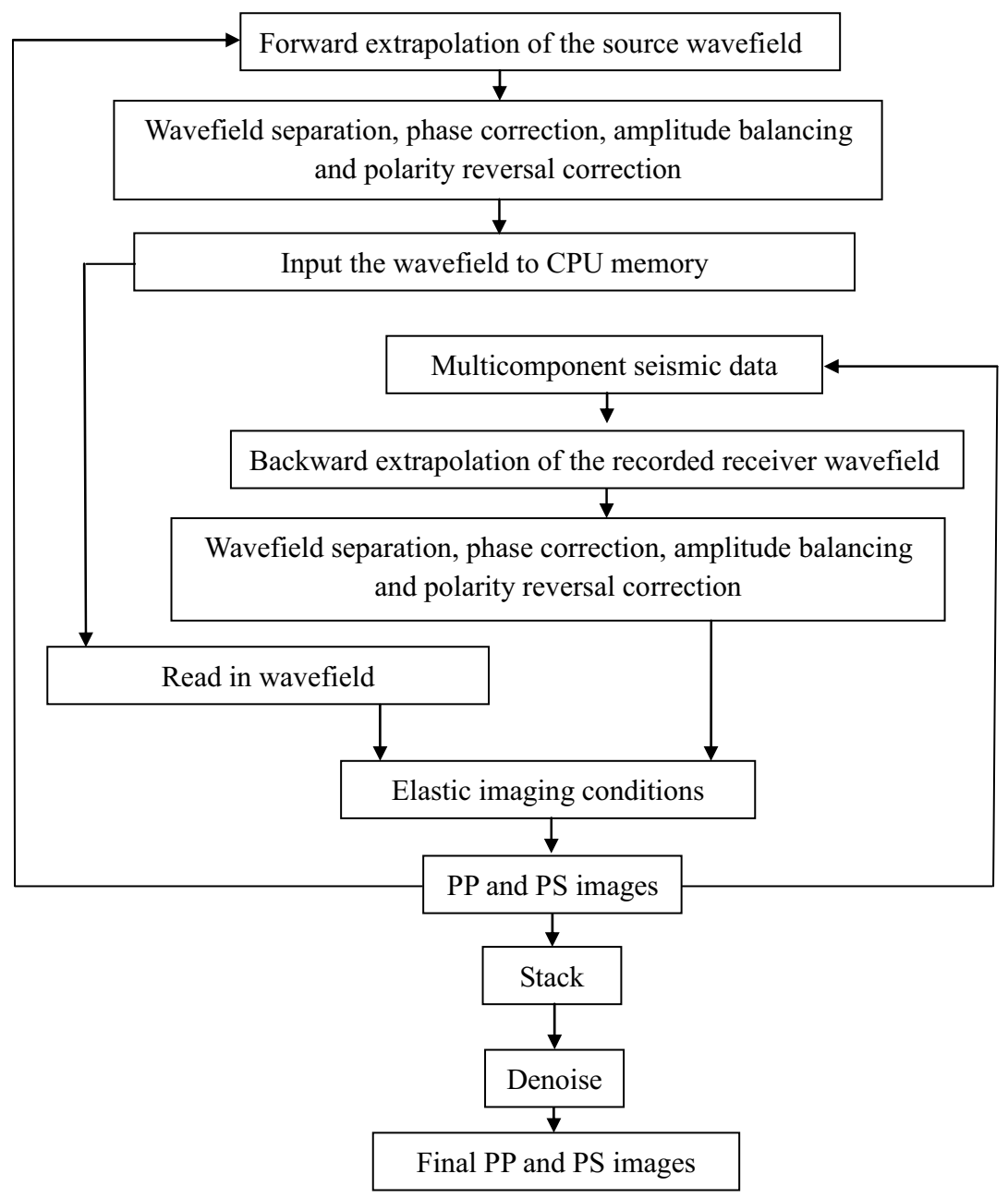

Fig. 2. Workflow chart for elastic prestack RTM.

The pure P-waves are represented with the divergence of the wavefield,

$$
P=\frac{\partial v_{x}}{\partial x}+\frac{\partial v_{z}}{\partial z} .
$$

The pure S-waves are represented with the curl of the wavefield,

$$
S=\frac{\partial v_{z}}{\partial x}-\frac{\partial v_{x}}{\partial z} .
$$


We can use the high-order SFD method to compute spatial derivatives for Eqs. 13 and 14.

The phase and amplitude of P-waves and S-waves separated by Helmholtz decomposition have changed, so we need to correct the phase and amplitude for the wavefield. Here, we adopt the method proposed by Sun et al. $(2001,2011)$ to solve this issue: Implementing the phase correction by Hilbert transform for separated P- and S-waves; and performing the amplitude balancing by taking the ratio of the P-wave velocity to the S-wave velocity times the S-wave amplitude.

Additionally, an S-image polarity reversal occurs because the S-wave changes its polarity when crossing normal incidence (Balch and Erdemir 1994). So the polarities must be corrected before applying the imaging conditions. Sun and McMechan (2001) specifically discussed the problem of polarity reversal for converted S-wave, and proposed that the polarity reversal could be corrected simply by multiplying the S-wave amplitudes by -1 on one side of the source position while keeping the other side unchanged after P-S wave separation. In this paper, we use this simple method (Sun and McMechan 2001) to perform polarity reversal correction in elastic RTM.

Yan and Sava (2008) proposed crosscorrelation imaging conditions to obtain elastic images with clear physical meanings. To reduce migration artifacts and improve image quality, Du et al. $(2012,2014)$ recommended source-normalized crosscorrelation imaging conditions. In this paper, the source-normalized crosscorrelation imaging conditions are adopted to perform the PP and PS imaging as follows (Du et al. 2012):

$$
\begin{aligned}
I_{\mathrm{PP}}(x, z) & =\frac{\sum_{\text {time }} S_{\mathrm{P}}(x, z, t) R_{\mathrm{P}}(x, z, t)}{\sum_{\text {time }} S_{\mathrm{P}}{ }^{2}(x, z, t)}, \\
I_{\mathrm{PS}}(x, z) & =\frac{\sum_{\text {time }} S_{\mathrm{P}}(x, z, t) R_{\mathrm{S}}(x, z, t)}{\sum_{\text {time }} S_{\mathrm{P}}{ }^{2}(x, z, t)},
\end{aligned}
$$

where $S(x, z, t)$ is the forward extrapolating source wavefield; $R(x, z, t)$ is the backward extrapolating receiver wavefield; the subscripts $\mathrm{P}$ and $\mathrm{S}$ denote $\mathrm{P}$ and $\mathrm{S}$ components, respectively; and $I_{\mathrm{pp}}(x, z)$ and $I_{\mathrm{ps}}(x, z)$ are PP- and PSmode migrated images, respectively. The cross-correlation imaging conditions usually produce low frequency migration noises. In this paper, we adopt a Laplace filtering presented by Zhang and Sun (2009) to suppress the low frequency noises. 


\section{EXAMPLES}

\subsection{Elastic prestack RTM for a groove model}

We use a groove model as shown in Fig. 3 to demonstrate the effects of elastic modeling and prestack RTM. The model is discretized into a 501 by 301 grid points, with a grid interval of $15 \mathrm{~m}$. The $\mathrm{P}$ - and S-wave velocities and the density for the upper layer are $2500 \mathrm{~m} / \mathrm{s}, 1500 \mathrm{~m} / \mathrm{s}$ and $2000 \mathrm{~kg}^{3} \mathrm{~m}^{-3}$, respectively, and for the lower layer are $2800 \mathrm{~m} / \mathrm{s}, 1800 \mathrm{~m} / \mathrm{s}$ and $2100 \mathrm{~kg}^{3} \mathrm{~m}^{-3}$, respectively. We use the sampling approximation SFD scheme with 2ndorder accuracy in time and 16th-order accuracy in space to generate a synthetic multicomponent data on the groove model. We choose a Ricker wavelet with a peak frequency of $25 \mathrm{~Hz}$ as the compressional source function. There are 42 sources in total, and the sources are placed at a horizontal interval of $150 \mathrm{~m}$, the first source being located at $(225 \mathrm{~m}, 30 \mathrm{~m})$. The receivers are distributed on the surface, with an interval of $15 \mathrm{~m}$. The sample interval is $0.001 \mathrm{~s}$ with a total propagation time of $5.0 \mathrm{~s}$. We perform forward and backward wavefield extrapolations for the elastic prestack RTM using the SFD methods with 2nd-order accuracy in time and 8th-order accuracy in space. Here, the perfectly matched layer (PML) absorbing boundary condition (Bérenger 1994) is used to reduce unwanted reflections from artificial boundaries.

Figures 4 and 5 show forward elastic wavefield snapshots at $t=1.35 \mathrm{~s}$ computed with the Taylor-series expansion SFD and the sampling approximation SFD schemes, respectively, when the source is located at $(3525 \mathrm{~m}$, $30 \mathrm{~m}$ ). Because we use an explosive source, the snapshots contain a mix of $\mathrm{P}$ - and S-wave modes, as can be seen form the $x$ and $z$ components. Furthermore, as highlighted by the white arrows, the numerical dispersion is very serious in the snapshots computed by the Taylor-series expansion SFD scheme. However, there is very small numerical dispersion in the snapshots

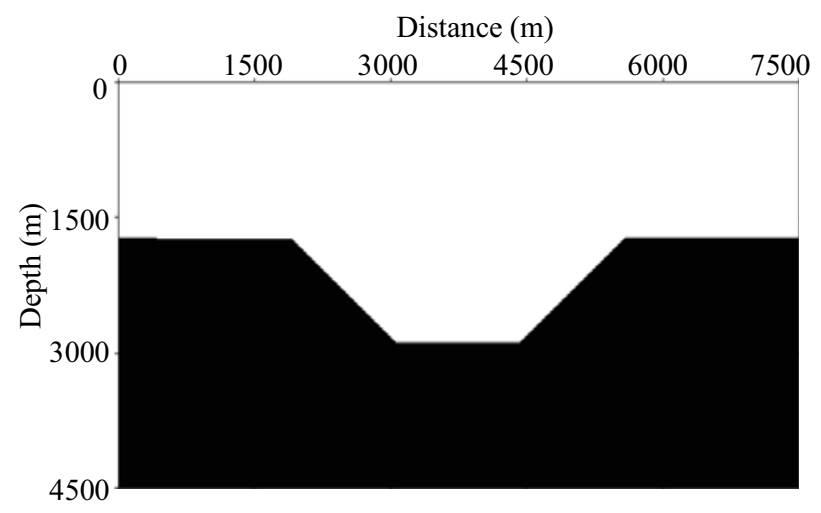

Fig. 3. Groove model. 

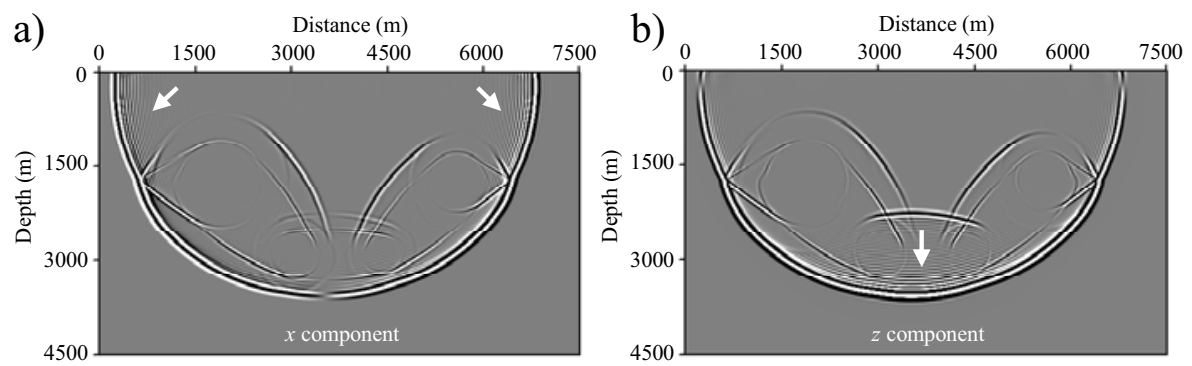

Fig. 4. Forward extrapolating wavefield snapshots computed by the Taylor-series expansion SFD scheme: (a) $x$ component, (b) $z$ component.
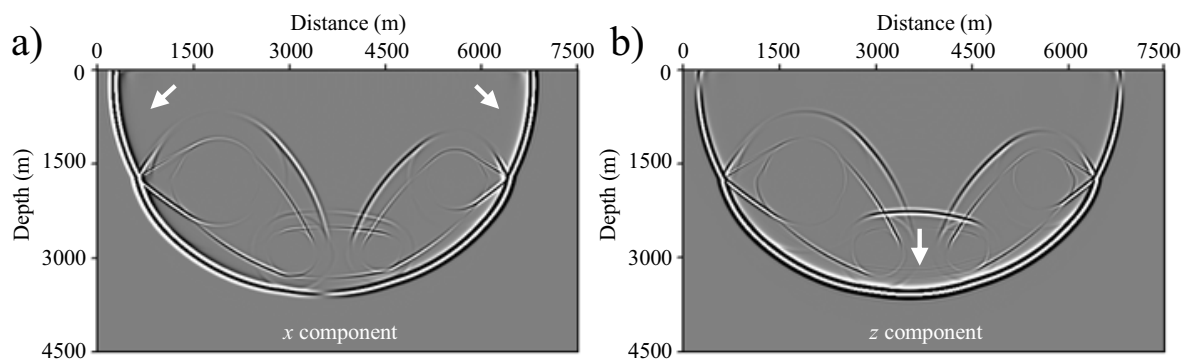

Fig. 5. Forward extrapolating wavefield snapshots computed by the sampling approximation SFD scheme: (a) $x$ component, (b) $z$ component.

computed by the sampling approximation SFD scheme, which further demonstrates that the sampling approximation SFD scheme has better accuracy in solving elastic wave equations.

We implement elastic prestack RTM using the Taylor-series expansion SFD scheme and the sampling approximation SFD scheme, respectively. Figures 6 and 7 show the final image results including PP images and PS images. Comparing Figs. 6 and 7, the differences among images generated by the Taylor-series expansion SFD scheme and the sampling approximation SFD scheme are obvious. And it can be observed that the events in Fig. 7 are clearer and more focused (highlighted by the white arrows). However, some artifacts along the reflector interfaces in the images migrated by the Taylorseries expansion SFD scheme are very serious (highlighted by the white arrows in Fig. 6), particularly for the PS image in Fig. 6b (highlighted by the white arrows), which results from numerical dispersion effects in the wave extrapolation. The serious dispersion in the extrapolating wavefield contaminates the images. Therefore, the numerical tests indicate the groove 


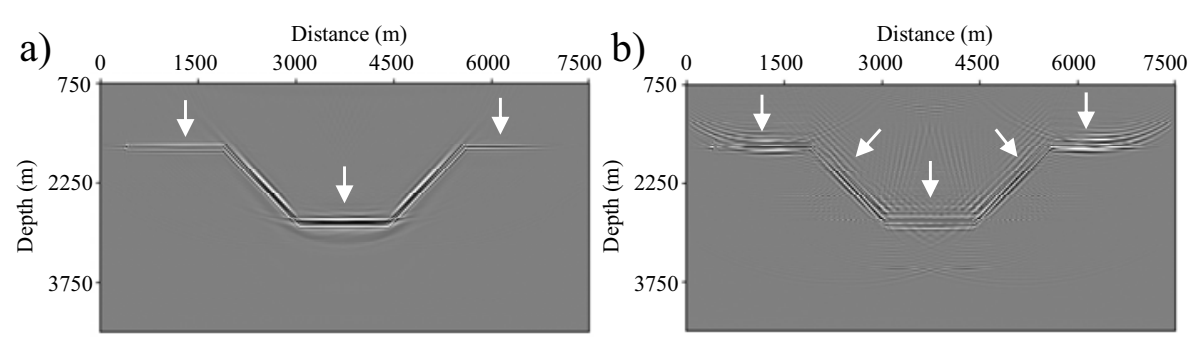

Fig. 6. Final PP and PS images migrated by the Taylor-series expansion SFD scheme for the fault model: (a) PP image, (b) PS image.

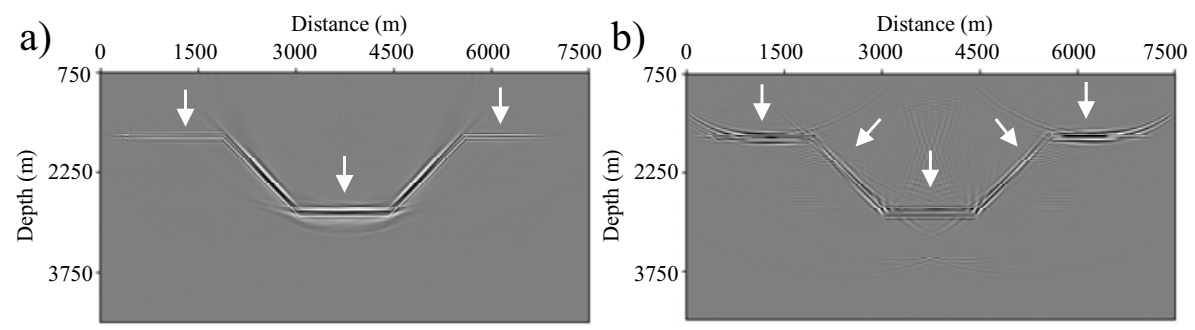

Fig. 7. Final PP and PS images migrated by the sampling approximation SFD scheme for the fault model: (a) PP image, (b) PS image.

model is better imaged by the sampling approximation SFD scheme than the Taylor-series expansion SFD scheme.

\subsection{Elastic prestack RTM for a salt model}

To further demonstrate the imaging quality and examine the efficiency of difference scheme with adaptive variable difference operator lengths, we test the elastic prestack RTM algorithms on the modified 2D Society of Exploration Geophysicists/European Association of Geoscientists and Engineers (SEG/EAGE) salt model. The salt model has 600 (in $x$ ) by 200 (in depth) grid points, with grid intervals $20 \mathrm{~m}$ (in $x$ ) and $20 \mathrm{~m}$ (in depth). The P-wave velocity information, as shown in Fig. 8, varies $2000 \mathrm{~m} / \mathrm{s}$ from $4981 \mathrm{~m} / \mathrm{s}$. The S-wave velocities $\left(v_{s}\right)$ are created from P-wave velocities $\left(v_{p}\right)$ following $v_{p} / v_{s}=1.7$. The density is constant. We simulate a synthetic multicomponent data set which has 75 shots with a $80 \mathrm{~m}$ shot spacing from the leftmost location $(3000 \mathrm{~m}, 40 \mathrm{~m})$ using the sampling approximation SFD scheme with 2 nd-order accuracy in time and 16th-order accuracy in space. The compressional source function is represented by a Ricker wavelet with a peak frequency of $30 \mathrm{~Hz}$. Each shot is recorded by 300 receivers spaced $20 \mathrm{~m}$ apart. The time sampling is interval $0.001 \mathrm{~s}$ and the recording time is $5 \mathrm{~s}$. 


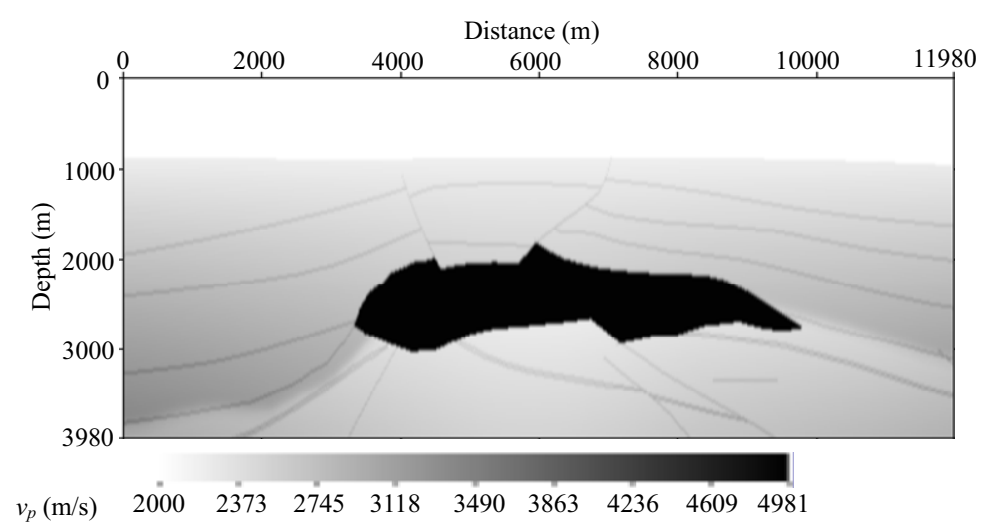

Fig. 8. P-wave velocity for the 2D salt model.

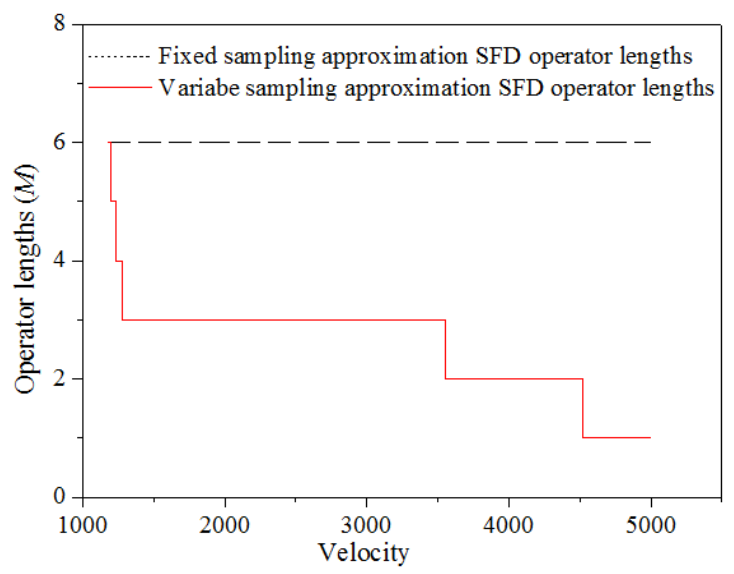

Fig. 9. Fixed and variable sampling approximation SFD operator lengths $M$ used in elastic RTM.

We implement the elastic prestack RTM using the Taylor-series expansion SFD scheme with the fixed difference operator lengths, the sampling approximation SFD scheme with the fixed difference operator lengths, and the sampling approximation SFD scheme with the adaptive variable difference operator lengths, respectively. Figure 9 shows the fixed and the variable sampling approximation SFD operator lengths $M$ used in elastic RTM. Figure 10 shows the final PP and PS images migrated by the Taylor-series expansion SFD scheme with fixed difference operator lengths. Figure 11 shows the final PP and PS images migrated by the sampling approximation SFD scheme with the fixed difference operator lengths. Figure 12 shows the final PP and PS images migrated by the sampling approximation SFD scheme with the adaptive variable difference operator lengths. 
a)

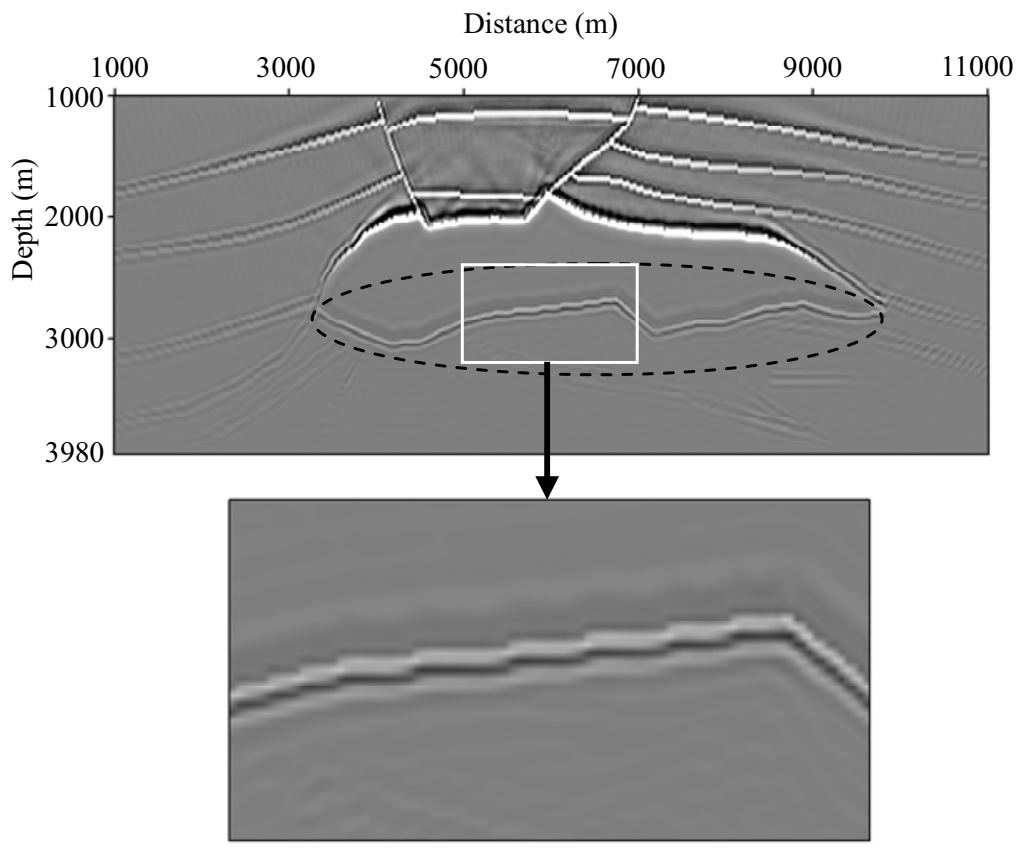

b)

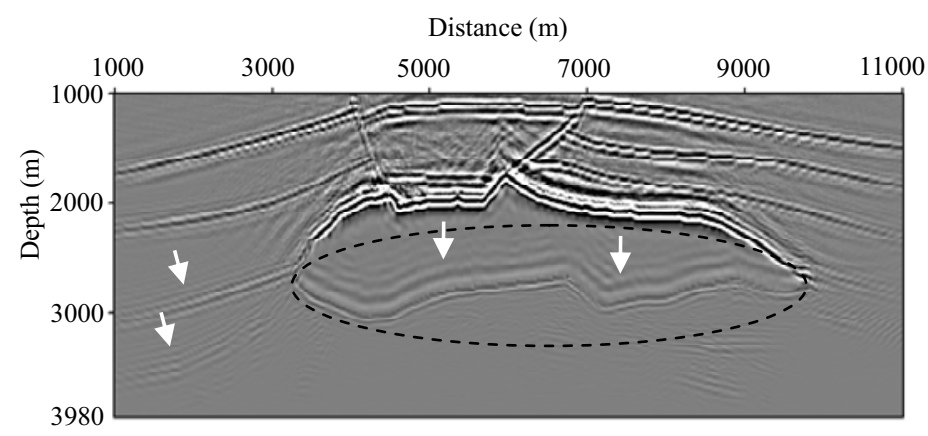

Fig. 10. Final PP and PS images migrated by the Taylor-series expansion SFD scheme with fixed difference operator lengths for the modified salt model: (a) PP image, (b) PS image.

From Figs. 10 and 11, we can see that the PP and PS images migrated by the sampling approximation SFD scheme are all very clear and their interfaces are well focused and imaged; however, the images migrated by the Taylor-series expansion SFD scheme are relatively fuzzy in regions along the salt marked by the black ellipses, and even some interfaces in the PS image have some artefacts like ripples (highlighted by the white arrows in 
a)

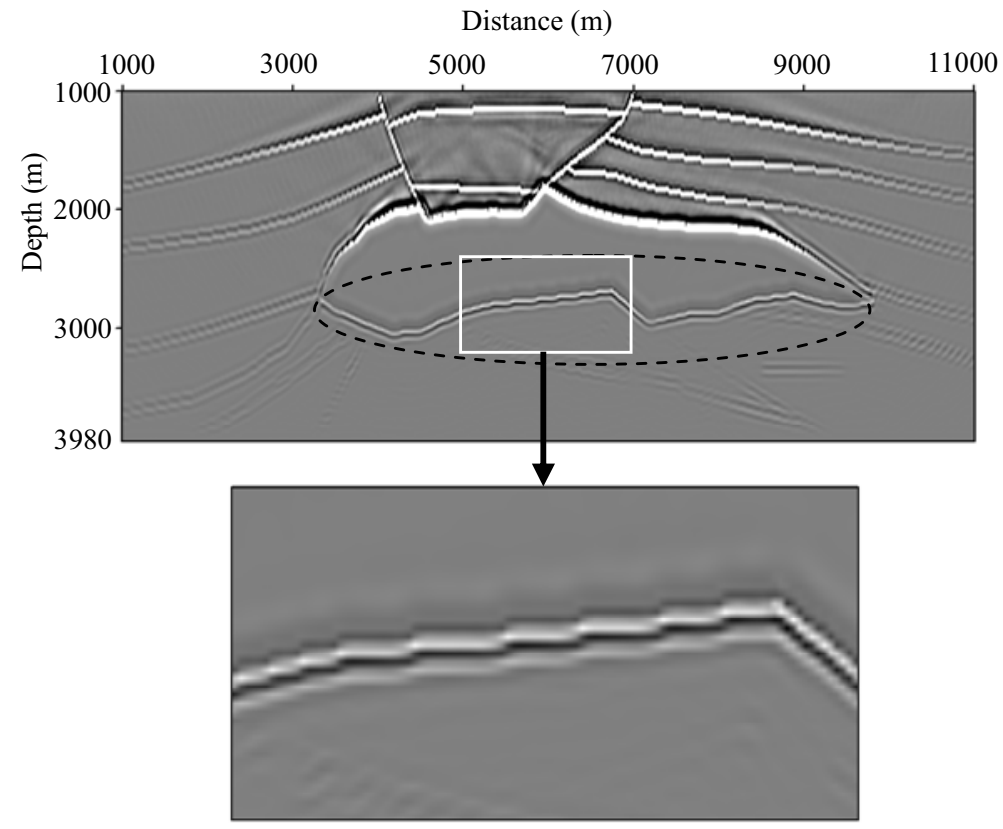

b)

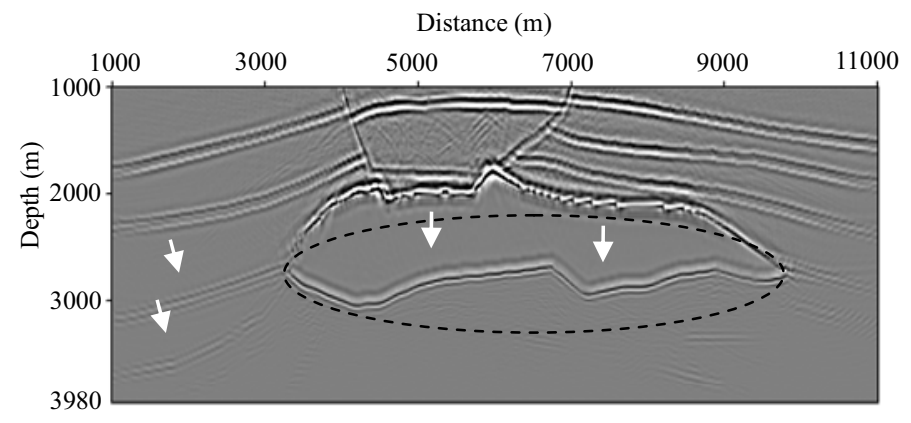

Fig. 11. Final PP and PS images migrated by the sampling approximation SFD scheme with fixed difference operator lengths for the modified salt model: (a) PP image, (b) PS image.

Fig. 10b). Comparing Figs. 11 and 12, we can hardly observe any difference and find that the images migrated by the sampling approximation SFD scheme with fixed and adaptive variable difference operator lengths have similar imaging accuracy, but their computational costs are very different. On the same computer (Dell OptiPlex 9020 with eight Intel Core $3.60 \mathrm{GHz}$ processors and 16.0 GB of memory), the computing time of the migration using fixed and adaptive variable difference operator lengths is $72142 \mathrm{~s}$ and 


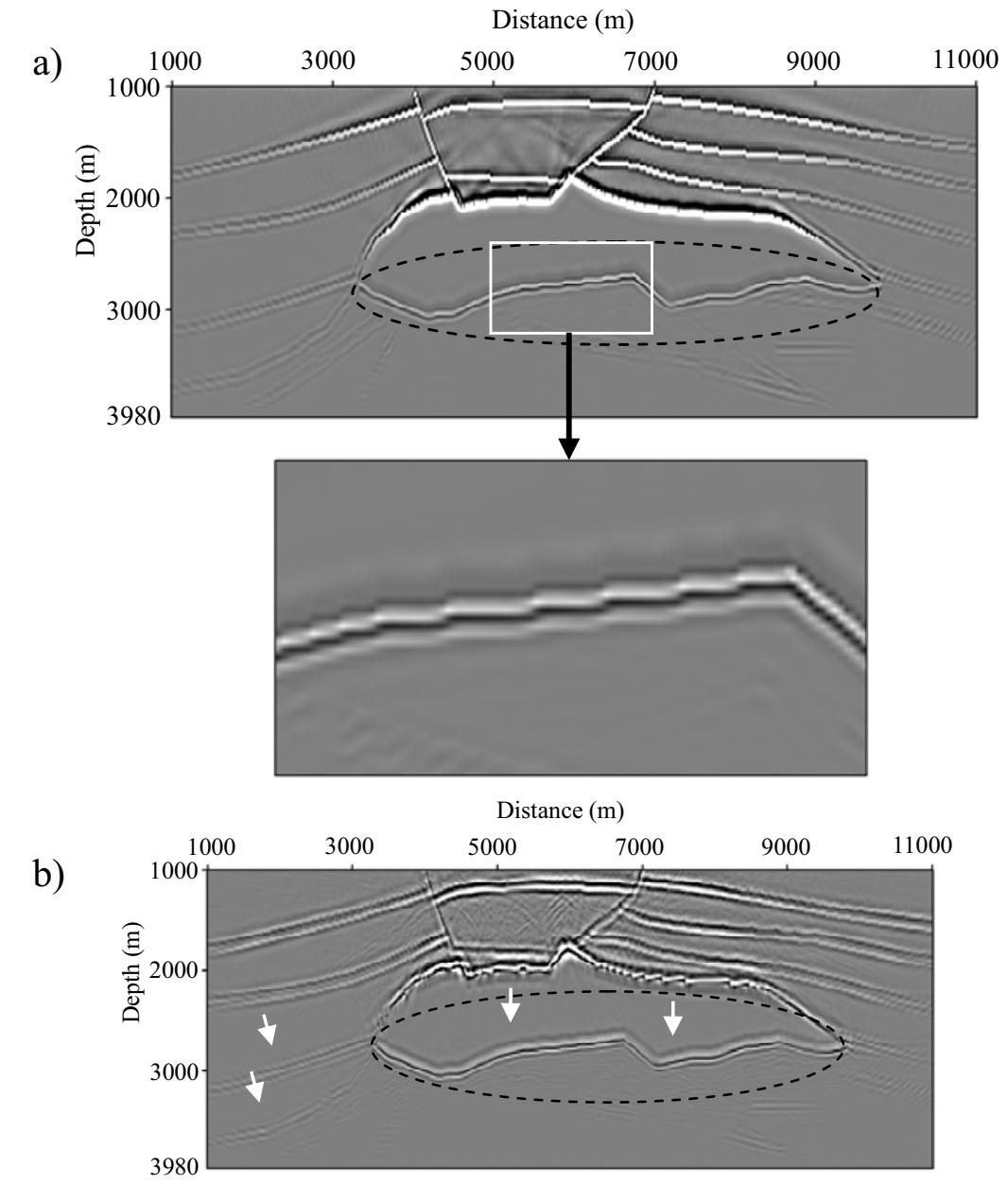

Fig. 12. Final PP and PS images migrated by the sampling approximation SFD scheme with adaptive variable difference operator lengths for the modified salt model: (a) PP image, (b) PS image.

$50191 \mathrm{~s}$, respectively. The application of adaptive variable difference operator lengths can improve computational efficiency compared with that of fixed difference operator lengths under the same imaging accuracy.

\subsection{Field data application}

In the final example, we use a field data set from the northeast China to test elastic RTM algorithm based on the sampling approximation SFD scheme with adaptive variable difference operator lengths, and compare it with that 

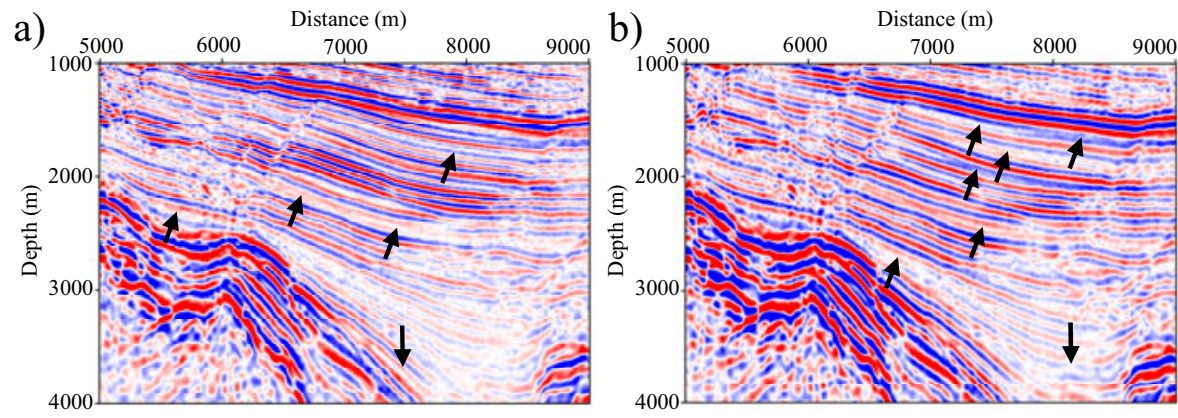

Fig. 13. PP and PS images migrated by the Taylor-series expansion SFD scheme with fixed difference operator lengths for the field data: (a) PP image, (b) PS image.
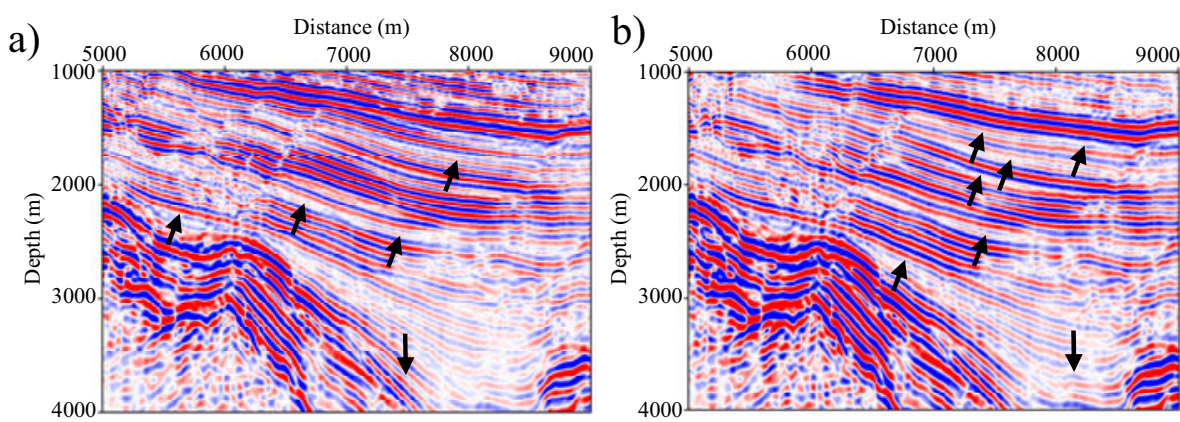

Fig. 14. PP and PS images migrated by the sampling approximation SFD scheme with adaptive variable difference operator lengths for the field data: (a) PP image, (b) PS image.

based on Taylor-series expansion SFD scheme. Figure 13 displays the migrated PP and PS images using the Taylor-series expansion SFD scheme with fixed difference operator lengths. Figure 14 displays the migrated PP and PS images using the sampling approximation SFD scheme with adaptive variable difference operator lengths. As shown in Figs. 13 and 14, two elastic RTM algorithms produce similar images, except in the areas marked by the black arrows. Some fuzzy events in the PP and PS images generated by the conventional elastic RTM algorithm based on Taylor-series expansion SFD scheme are visible (highlighted by the black arrows in Fig. 13a and b), which reduces the seismic imaging accuracy. In contrast, the events in the PP and PS images (highlighted by the black arrows in Fig. 14a and b) generated by the proposed elastic RTM algorithm are clearer and more focused. The field data test shows that the proposed RTM algorithm can produce better images than the conventional algorithm. Furthermore, this field data test also shows computational efficiency of the RTM with the adaptive variable 
difference operator lengths improves by about $28 \%$, compared with that with the fixed difference operator lengths.

\section{CONCLUSIONS}

We have put forward and used an efficient SFD scheme based on a sampling approximation method with adaptive variable difference operator lengths to implement elastic prestack RTM. The sampling approximation SFD scheme is used to solve elastic wave equations for forward and backward wavefield extrapolations. Numerical dispersion analysis and the wavefield extrapolation results show that the sampling approximation SFD scheme has greater accuracy than the Taylor-series expansion SFD scheme. We tested the elastic prestack RTM algorithm based on the sampling approximation SFD scheme with adaptive variable difference operator lengths. Experiments on two theoretical models and a field data set demonstrate that the elastic prestack RTM using the sampling approximation SFD scheme can generate better images than that using the Taylor-series expansion SFD scheme, particularly for PS images. Compared with the conventional fixed difference operator lengths, the application of the adaptive variable difference operator lengths can effectively improve computational efficiency in elastic prestack RTM.

Acknowledgements. This work is supported by National Natural Science Foundation of China (Grant No. 41404112), the International Postdoctoral Exchange Fellowship Program (the Office of China Postdoctoral Council) (Grant No. 20140047), and the Edinburgh Anisotropy Project (EAP) of the British Geological Survey.

\section{References}

Balch, A.H., and C. Erdemir (1994), Sign-change correction for prestack migration of P-S converted wave reflections, Geophys. Prospect. 42, 6, 637-663, DOI: 10.1111/j.1365-2478.1994.tb00233.x.

Baysal, E., D.D. Kosloff, and J.W.C. Sherwood (1983), Reverse time migration, Geophysics 48, 11, 1514-1524, DOI: 10.1190/1.1441434.

Bérenger, J.P. (1994), A perfectly matched layer for the absorption of electromagnetic waves, J. Comput. Phys. 114, 2, 185-200, DOI: 10.1006/jcph.1994. 1159.

Chang, W.F., and G.A. McMechan (1987), Elastic reverse-time migration, Geophysics 52, 10, 1365-1375, DOI: 10.1190/1.1442249.

Chang, W.F., and G.A. McMechan (1994), 3D elastic prestack reverse-time depth migration, Geophysics 59, 4, 597-609, DOI: 10.1190/1.1443620. 
Chung, W., S. Pyun, H.S. Bae, C. Shin, and K.J. Marfurt (2012), Implementation of elastic reverse-time migration using wavefield separation in the frequency domain, Geophys. J. Int. 189, 3, 1611-1625, DOI: 10.1111/j.1365246X.2012.05431.x.

Dablain, M.A. (1986), The application of high-order differencing to the scalar wave equation, Geophysics 51, 1, 54-66, DOI: 10.1190/1.1442040.

Dellinger, J., and J. Etgen (1990), Wave-field separation in two-dimensional anisotropic media, Geophysics 55, 7, 914-919, DOI: 10.1190/1.1442906.

Dong, L.G., Z.T. Ma, J.Z. Cao, H.Z. Wang, J.H. Gao, B. Lei, and S.Y. Xu (2000), A staggered-grid high-order difference method of one-order elastic wave equation, Chin. J. Geophys. 43, 411-419 (in Chinese).

Du, Q., Y. Zhu, and J. Ba (2012), Polarity reversal correction for elastic reverse time migration, Geophysics 77, 2, S31-S41, DOI: 10.1190/geo2011-0348.1.

Du, Q., X. Gong, M. Zhang, Y. Zhu, and G. Fang (2014), 3D PS-wave imaging with elastic reverse-time migration, Geophysics 79, 5, S173-S184, DOI: 10.1190/geo2013-0253.1.

Gray, S.H., J. Etgen, J. Dellinger, and D. Whitmore (2001), Seismic migration problems and solutions, Geophysics 66, 5, 1622-1640, DOI: 10.1190/1.1487107.

Hokstad, K. (2000), Multicomponent Kirchhoff migration, Geophysics 65, 3, 861873, DOI: $10.1190 / 1.1444783$.

Kindelan, M., A. Kamel, and P. Sguazzero (1990), On the construction and efficiency of staggered numerical differentiators for the wave equation, Geophysics 55, 1, 107-110, DOI: 10.1190/1.1442763.

Kuo, J.T., and T.F. Dai (1984), Kirchhoff elastic wave migration for the case of noncoincident source and receiver, Geophysics 49, 8, 1223-1238, DOI: 10.1190/1.1441751.

Li, J., D. Yang, and F. Liu (2013), An efficient reverse time migration method using local nearly analytic discrete operator, Geophysics 78, 1, S15-S23, DOI: 10.1190/geo2012-0247.1.

Li, J., M. Fehler, D. Yang, and X. Huang (2015), 3D weak-dispersion reverse time migration using a stereo-modeling operator, Geophysics 80, 1, S19-S30, DOI: 10.1190/geo2013-0472.1.

Liu, F., G. Zhang, S.A. Morton, and J.P. Leveille (2009), An optimized wave equation for seismic modeling and reverse time migration, Geophysics 74, 6, WCA153-WCA158, DOI: 10.1190/1.3223678.

Liu, Y. (2014), Optimal staggered-grid finite-difference schemes based on leastsquares for wave equation modeling, Geophys. J. Int. 197, 2, 1033-1047, DOI: $10.1093 / \mathrm{gji} / \mathrm{ggu} 032$.

Liu, Y., and M.K. Sen (2009), An implicit staggered-grid finite-difference method for seismic modeling, Geophys. J. Int. 179, 1, 459-474, DOI: 10.1111/ j.1365-246X.2009.04305.x. 
Liu, Y., and M.K. Sen (2011a), Finite-difference modeling with adaptive variablelength spatial operators, Geophysics 76, 4, T79-T89, DOI: 10.1190/ 1.3587223 .

Liu, Y., and M.K. Sen (2011b), Scalar wave equation modeling with time-space domain dispersion-relation-based staggered-grid finite-difference schemes, Bull. Seismol. Soc. Am. 101, 1, 141-159, DOI: 10.1785/0120100041.

Pei, Z. (2004), Numerical modeling using staggered-grid high order finite difference of elastic wave equation on arbitrary relief surface, Oil Geophys. Prospect. 39, 629-634 (in Chinese).

Sun, R., and G.A. McMechan (2001), Scalar reverse-time depth migration of prestack elastic seismic data, Geophysics 66, 5, 1519-1527, DOI: 10.1190/ 1.1487098 .

Sun, R., G.A. McMechan, and H.-H. Chuang (2011), Amplitude balancing in separating P- and S-waves in 2D and 3D elastic seismic data, Geophysics 76, 3, S103-S113, DOI: 10.1190/1.3555529.

Virieux, J. (1986), P-SV wave propagation in heterogeneous media: Velocity stress finite difference method, Geophysics 51, 4, 889-901, DOI: 10.1190/ 1.1442147 .

Whitmore, D. (1983), Iterative depth migration by backward time propagation. In: 53rd Annual International Meeting, SEG, Expanded Abstracts, 382-385.

Yan, H., Y. Liu, and H. Liu (2013), Elastic prestack reverse-time migration using the time-space domain high-order staggered-grid finite-difference method. In: 83rd Annual International Meeting, SEG, Expanded Abstracts, 40054009.

Yan, H., L. Yang, and H. Liu (2015), Acoustic reverse-time migration using optimal staggered-grid finite-difference operator based on least squares, Acta Geophys. 63, 3, 715-734, DOI: 10.2478/s11600-014-0259-9.

Yan, J., and P. Sava (2008), Isotropic angle-domain elastic reverse-time migration, Geophysics 73, 6, S229-S239, DOI: 10.1190/1.2981241.

Yan, R., and X.B. Xie (2012), An angle-domain imaging condition for elastic reverse time migration and its application to angle gather extraction, Geophysics 77, 5, S105-S115, DOI: 10.1190/geo2011-0455.1.

Yang, L., H. Yan, and H. Liu (2014), Least squares staggered-grid finite-difference for elastic wave modeling, Explor. Geophys. 45, 4, 255-260, DOI: 10.1071/ EG13087.

Yang, L., H. Yan, and H. Liu (2015), Optimal rotated staggered-grid finitedifference schemes for elastic wave modeling in TTI media, J. Appl. Geophys. 122, 40-52, DOI: 10.1016/j.jappgeo.2015.08.007.

Zhang, Y., and J. Sun (2009), Practical issues of reverse time migration: True amplitude gathers, noise removal and harmonic-source encoding, First Break 26, $19-25$. 
Zhou, H., and G. Zhang (2011), Prefactored optimized compact finite difference schemes for second spatial derivatives, Geophysics 76, 5, WB87-WB95, DOI: 10.1190/geo2011-0048.1.

Received 26 May 2015

Received in revised form 8 February 2016

Accepted 18 February 2016 